ISSN: 2354 - 1431

http://tckh.daihoctantrao.edu.vn/

\title{
Tương tác của các boson chuẩn trong mô hình Zee
}

Hà Thanh Hùng ${ }^{a, *}$

a Trường Đại học Sư phạm Hà Nội 2

\section{Article info}

Recieved:

03/7/2017

Accepted:

03/8/2017

Keywords:

Zee model;

gauge boson;

lepton flavor violating;

Higgs decays to two photon;

etc

\begin{abstract}
By adding scalars, the Zee model, as standard model byond, has generated masses for the neutrinos. Due to the addition of new scalars, the mass spectrum and physical states of the particles are different from ones of the standard model. Using general Lagrangian, the interactions of gauge bosons with fermions and Higgs bosons are given. These vertices are the basis for the study of decay processes such as: lepton flavor violating Higgs decays, Higgs decays to two photon.
\end{abstract}

\section{Giới thiệu}

Sự hoạt động của các máy gia tốc hạt với năng lượng ngày càng cao đã cung cấp nhiều số liệu thực nghiệm cho ngành Vật lý năng lượng cao [3,4]. Một trong những kết quả thành công rút ra từ các số liệu thực nghiệm đó là khẳng định sự tồn tại chắc chắn của Higgs boson. Đây cũng là phần tiên đoán cuối cùng của mô hình chuẩn cần được kiểm chứng. Sự thành công của mô hình chuẩn đã được kiểm chứng qua các số liệu thực nghiệm, tuy nhiên cũng còn nhiều hạn chế chưa giải quyết được. Một trong các hạn chế lớn nhất của mô hình chuẩn là giải quyết khối lượng và sự dao động của neutrino. Mô hình Zee được đề xuất như một hướng mở rộng của mô hình chuẩn cho việc sinh khối lượng neutrino ở các bổ đính bậc cao [2]. Các mô hình mở rộng mô hình chuẩn cũng đem lại nhiều hiệu quả Vật lý mới cần nghiên cứu, các Vật lý mới này được đưa ra từ các kênh rã vi phạm số lepton thế hệ [5,9], từ các kênh rã của các boson trung hòa ra các photon ...[6,8]. Đây là các kênh rã hiếm và hiện nay đang rất được quan tâm nghiên cứu, đóng góp của các boson chuẩn trong các kênh rã này là phần quan trọng không thể thiếu, nhất là ở bậc một vòng.

Bài báo này nghiên cứu tương tác của các boson chuẩn trong mô hình Zee xuất phát từ Lagrangian toàn phần. Các đỉnh tương tác của các boson chuẩn với các fermion và các Higgs boson được đưa ra. Đây là cơ sở để nghiên cứu các kênh rã Higgs và tìm ra các Vật lý mới trong mô hình Zee.

\section{Nội dung}

\subsection{Các boson chuẩn trong mô hình Zee}

Mô hình Zee được đưa ra như là một hướng mở rộng của mô hình chuẩn để giải quyết khối lượng của neutrino. Trong mô hình này, khối lượng các neutrino được sinh ra ở các bổ đính bậc cao do sự đóng góp của các trường vô hướng mới thêm vào so với mô hình chuẩn. Các hạt mới thêm vào bao gồm một lưỡng tuyến Higgs giống như mô hình chuẩn và một Higgs mang điện thuộc biểu diễn đơn tuyến của nhóm $S U(2)_{L}$. Các hạt trong mô hình được sắp xếp như sau [2]:

Lepton: Các hạt phân cực trái được xếp vào lưỡng tuyến, còn các hạt phân cực phải xếp vào đơn tuyến của nhóm $S U(2)_{L}$

$\psi_{a L}=\left(v_{a}, e_{a}\right)_{L}^{T} \sim(1,2,-1), e_{\mathrm{aR}} \sim(1,1,-2)$

Quark: Các hạt phân cực trái được xếp vào lưỡng tuyến, còn các hạt phân cực phải xếp vào đơn tuyến của nhóm $S U(2)_{L}$

$Q_{a L}=\left(u_{a L}, d_{a L}\right)^{T} \sim(3,2,1 / 3) ; u_{a R} \sim(3,1,4 / 3) ; d_{a R} \sim(3,1,4 / 3)$

Với $a=1,2,3$ tương ứng là chỉ số thế hệ, còn bộ ba số trong ngoặc đơn tương ứng là số lượng tử của các nhóm $S U(3)_{C}, S U(2)_{L}, U(1)_{Y}$ 
Vô hướng: Trong mô hình Zee có hai lưỡng tuyến của nhóm $S U(2)_{L}$ và một Higgs mang điện đơn. Cụ thể là:

$\phi_{1}=\left(\phi_{1}^{+}, \phi_{1}^{0}\right)^{T} \sim(1,2,1), \phi_{2}=\left(\phi_{2}^{+}, \phi_{2}^{0}\right)^{T} \sim(1,2,1), h^{+} \sim(1,1,2)$

Thành phần lưỡng tuyến được biểu diễn theo giá trị trung bình chân không v1, v2 như sau:

$\phi_{1}=\left(\begin{array}{c}\phi_{1}^{+} \\ \frac{1}{\sqrt{2}}\left(\mathrm{v}_{1}+\phi_{1 R}^{0}+i \phi_{1 I}^{0}\right)\end{array}\right) ; \phi_{2}=\left(\begin{array}{c}\phi_{2}^{+} \\ \frac{1}{\sqrt{2}}\left(\mathrm{v}_{2}+\phi_{2 R}^{0}+i \phi_{2 I}^{0}\right)\end{array}\right)(4)$

Tương tự như mô hình chuẩn, các boson chuẩn được xác định từ đạo hàm hiệp biến [1].

$D_{\mu} \phi=\left(\partial_{\mu}-\frac{i}{2} \mathrm{gA}_{\mu a} \sigma^{a}-\frac{i}{2} \mathrm{~g}^{\prime} \mathrm{B}_{\mu}\right) \phi=\left(\partial_{\mu}-i P_{\mu}^{\phi}\right) \phi(5)$

Trong đó $P_{\mu}^{\phi}$ được biểu thị theo các trường vật lý như sau:

$P_{\mu}^{\phi}=\frac{\sqrt{2} g}{2}\left(\begin{array}{cc}\frac{\left(1-2 \mathrm{~s}_{\square}^{2}\right)}{\sqrt{2} c_{\square}} Z_{\mu}+\sqrt{2} s_{\square} A_{\mu} & \square_{\mu}^{+} \\ \square{ }_{\mu}^{-} & -\frac{Z_{\mu}}{\sqrt{2} c_{\square}}\end{array}\right)$

Tương ứng với mối liên hệ giữa các trường vật lý và các trường chuẩn là:

$\square^{\mu \pm}=\frac{1}{\sqrt{2}}\left(\mathrm{~A}_{1}^{\mu} \mp \mathrm{iA}_{2}^{\mu}\right), Z_{\mu}=c_{\square} A_{\mu}^{3}-s_{\square} B_{\mu}$,

$A_{\mu}=s_{\square} A_{\mu}^{3}+c_{\square} B_{\mu}$

Với $\mathrm{s}_{\mathrm{u}}=\sin \theta_{\mathrm{\square}}, c_{\mathrm{\square}}=\cos \theta_{\mathrm{\square}}, \tan \theta_{\mathrm{\square}}=\frac{g^{\prime}}{g}$

Trong bốn trường chuẩn này, có hai trường mang điện là $\square^{ \pm}$và hai trường trung hòa $Z_{\mu}, A_{\mu}$, trường $A_{\mu}$ ứng với photon.

Khối lượng của các boson chuẩn trong mô hình Zee được xác định từ số hạng động năng của trường Higgs tương ứng với phần có trung bình chân không.

$L_{k i n}^{\text {Higgs }}=\sum_{S}\left(D_{\mu}\langle S\rangle\right)^{+}\left(D_{\mu}\langle S\rangle\right)$

Với $S=\phi_{1}, \phi_{2}, h^{+}$

Bằng cách đặt $v_{1}=v \sin \beta \equiv v s_{\beta}, v_{2}=v \cos \beta \equiv v c_{\beta}$, từ (8) chúng ta thu được các khối lượng: $m_{\square^{ \pm}}=\frac{g v}{2}, m_{Z}=\frac{g v}{2 \sqrt{2} c_{\square}} ; m_{A}=0$

2.2. Tương tác của các boson chuẩn trong mô hình Zee

Do việc thêm vào các vô hướng mới, Lagrangian tổng quát trong mô hình Zee là [2]:

$\mathcal{L}_{\text {tot }}=\mathcal{L}_{S M}+\mathcal{L}_{\text {Zee }}$

Trong đó:

$\mathcal{L}_{S M}=\sum_{f} \bar{\psi} i \gamma^{\mu} D_{\mu} \psi+\sum_{S}\left(D_{\mu} S\right)^{+}\left(D_{\mu} S\right)-\frac{1}{4} G_{i \mu \nu} G_{i}^{u v}-\frac{1}{4} A_{\mu \nu} A_{i}^{\mu v}-\frac{1}{4} B_{\mu \nu} B^{\mu \nu}+\mathcal{L}_{Y}-V$ là Lagragian của mô hình chuẩn và $f$ là tập của tất cả các đa tuyến fermion, $S$ là tập của tất cả các đa tuyến vô hướng có trong mô hình.

Các tương tác mới do sự xuất hiện của các hạt mới trong mô hình Zee được thể hiện ở số hạng sau:

$\mathcal{L}_{\text {Zee }}=\sum_{a, b=e, \mu, \tau} f_{a b} \psi_{a L}^{T} C\left(i \sigma_{2}\right) \psi_{b L} h^{+}+h . c$

Thế Higgs tổng quát được viết bởi $[5,9]$, cụ thể:

$V=\mu_{1}^{2} \phi_{1}^{+} \phi_{1}+\mu_{2}^{2} \phi_{2}^{+} \phi_{2}+\frac{\lambda_{1}}{2}\left(\phi_{1}^{+} \phi_{1}\right)^{2}+\frac{\lambda_{2}}{2}\left(\phi_{2}^{+} \phi_{2}\right)^{2}$

$+\lambda_{3}\left(\phi_{1}^{+} \phi_{1}\right)\left(\phi_{2}^{+} \phi_{2}\right)+\lambda_{4}\left(\phi_{1}^{+} \phi_{2}\right)\left(\phi_{2}^{+} \phi_{1}\right)+\frac{\lambda_{5}}{2}\left[\left(\phi_{1}^{+} \phi_{2}\right)^{2}+h . c\right]$

$+\mu_{h}^{2} h^{+} h^{-}+\lambda_{h}\left(h^{+} h^{-}\right)^{2}+\lambda_{6}\left(h^{+} h^{-}\right)\left(\phi_{1}^{+} \phi_{1}\right)$

$+\lambda_{7}\left(h^{+} h^{-}\right)\left(\phi_{2}^{+} \phi_{2}\right)+\mu\left(\phi_{1}^{+} i \sigma_{2} \phi_{2}^{*} h^{+}+\right.$h.c. $)$

$+\left(\phi_{1}^{+} \phi_{2}+\phi_{2}^{+} \phi_{1}\right)\left(-\mu_{12}^{2}+\lambda_{8} \phi_{1}^{+} \phi_{1}+\lambda_{9} \phi_{2}^{+} \phi_{2}+\lambda_{10} h^{+} h^{-}\right)$

Để xác định tương tác của các boson chuẩn với các fermion và các Higgs boson, chúng ta cần xác định trạng thái vật lý của các boson. Khác với mô hình chuẩn, do sự xuất hiện thêm một lưỡng tuyến và một điện tích đơn nên trong mô hình Zee sẽ có hai điện tích đơn $\left(\mathrm{H}_{1}^{+}, \mathrm{H}_{2}^{+}\right)$, hai Higgs trung hòa $(H, h)$, trong đó $h$ tương ứng với Higgs trung hòa trong mô hình chuẩn. Các mối liên hệ đặc trưng cho mô hình Zee được đưa ra như sau:

Kí hiệu các thành phần của Higgs bosson mang điện trong hai lưỡng tuyến là

$\phi^{ \pm} \equiv \cos \beta \phi_{1}^{ \pm}-\sin \beta \phi_{2}^{ \pm}$

Trong đó, $t_{\beta} \equiv \tan \beta=\frac{\mathrm{v}_{1}}{\mathrm{v}_{2}}$

Các Goldstone bosson $\omega^{ \pm}$và $\mathrm{z}$ tương ứng với gauge bosson $\mathrm{W}^{ \pm}$và $\mathrm{Z}$ được cho bởi [2]:

$\left(\begin{array}{c}\phi^{ \pm} \\ \omega^{ \pm}\end{array}\right)=\left(\begin{array}{cc}\cos \beta & -\sin \beta \\ \sin \beta & \cos \beta\end{array}\right)\left(\begin{array}{c}\phi_{1}^{ \pm} \\ \phi_{2}^{ \pm}\end{array}\right)$;

$\left(\begin{array}{c}A \\ z\end{array}\right)=\left(\begin{array}{cc}\cos \beta & -\sin \beta \\ \sin \beta & \cos \beta\end{array}\right)\left(\begin{array}{l}\phi_{I 1}^{0} \\ \phi_{I 2}^{0}\end{array}\right)$

Trạng thái vật lý của Higgs bosson trung hòa và mang điện được đưa ra theo [5]

$\left(\begin{array}{l}H \\ h\end{array}\right)=\left(\begin{array}{cc}\cos \alpha & -\sin \alpha \\ \sin \alpha & \cos \alpha\end{array}\right)\left(\begin{array}{l}\phi_{R 1}^{0} \\ \phi_{R 2}^{0}\end{array}\right)$

$\left(\begin{array}{c}H_{1}^{+} \\ H_{2}^{+}\end{array}\right)=\left(\begin{array}{cc}\cos \theta_{z} & -\sin \theta_{z} \\ \sin \theta_{z} & \cos \theta_{z}\end{array}\right)\left(\begin{array}{l}h^{+} \\ \phi^{+}\end{array}\right)$;

Trong đó $\sin 2 \theta_{z}=\frac{2 \sqrt{2} \mu m_{\square}}{g\left(M_{1}^{2}-M_{2}^{2}\right)}$ với $M_{1,2}$ tương ứng là khối lượng của các Higgs mang điện $\left(H_{1,2}\right)$ và $m_{\square}$ là khối lượng của W-boson. 
- Tương tác của các boson chuẩn với các fermion:

Các tương tác được xác định từ số hạng Lagrangian động năng của các đa tuyến fermions sau:

$$
\mathcal{L}^{f}{ }_{k i n}=\sum_{f} \bar{\psi} i \gamma^{\mu} D_{\mu} \psi
$$

Các đỉnh tương tác được xác định thông qua các trạng thái vật lý. Tức là, khi đó chúng ta phải làm việc trong các hệ cơ sở mới mà ma trận khối lượng của các fermion có dạng chéo. Ma trận chuyển cơ sở cho các neutrino và các quark trong trường hợp này tương ứng là:

$$
v_{a}^{\prime}=U_{a b} v_{b}, q_{a}^{\prime}=V_{a b} q_{b}
$$

Đặc biệt, việc đưa vào ma trận $V_{a b}$ còn đảm bảo cho dòng trung hòa không bị thay đổi số vị (tích của nhóm $S U(2)_{L}$ ) phù hợp với các dữ liệu từ thực nghiệm.

Thay các phương trình (1), (2) vào (17) kết hợp với (18) ta có các đỉnh tương tác của các fermion với các boson chuẩn như bảng 1 :

Bảng 1: Các đỉnh tuoong tác của các fermion với các boson chuẩn

\begin{tabular}{|l|l|}
\hline Đỉnh & Hệ số \\
\hline $\bar{v} \square{ }_{\mu}{ }^{+} e_{b}$ & $\frac{i g}{\sqrt{2}} U_{a b}^{*} \gamma^{\mu} P_{L}$ \\
\hline $\bar{e} \square{ }_{\mu}{ }^{-} v_{b}$ & $\frac{i g}{\sqrt{2}} U_{a b} \gamma^{\mu} P_{L}$ \\
\hline $\bar{u} \square{ }_{\mu}^{+} d_{b}$ & $\frac{i g}{\sqrt{2}} V_{a b} \gamma^{\mu} P_{L}$ \\
\hline $\bar{d} \square_{\mu}{ }^{-} u_{b}$ & $\frac{i g}{\sqrt{2}} V^{*}{ }_{a b} \gamma^{\mu} P_{L}$ \\
\hline $\bar{v} Z_{\mu} v$ & $\frac{i g}{2 c_{\square}} \gamma^{\mu} P_{L}$ \\
\hline $\bar{e} Z_{\mu} e$ & $\frac{i g}{4 c_{\square}} \gamma^{\mu}\left[\left(-1+4 s_{\square}^{2}\right)+\gamma_{5}\right]$ \\
\hline $\bar{u} Z_{\mu} u$ & $\frac{i g}{4 c_{\square}} \gamma^{\mu}\left[\left(1-\frac{8 s_{\square}^{2}}{3}\right)+\gamma_{5}\right]$ \\
\hline $\bar{d} Z_{\mu} d$ & $\frac{i g}{4 c_{\square}} \gamma^{\mu}\left[\left(-1+\frac{4 s_{\square}^{2}}{3}\right)-\gamma_{5}\right]$ \\
\hline
\end{tabular}

- Tương tác của các boson chuẩn với các Higgs boson.

Ở dạng đầy đủ, số hạng động năng của các trường Higgs được thể hiện:

$\mathcal{L}^{H i g g s}{ }_{k i n}=\sum_{S}\left(D_{\mu} S\right)^{+}\left(D_{\mu} S\right)$

$=\sum_{S}\left[\left(\partial_{\mu} S\right)^{+}\left(\partial_{\mu} S\right)+\left(P_{\mu} S\right)^{+}\left(\partial_{\mu} S\right)+\left(\partial_{\mu} S\right)^{+}\left(P_{\mu} S\right)+\left(P_{\mu} S\right)^{+}\left(P_{\mu} S\right)\right]$

Các số hạng thứ 2 và thứ 3 của (19) cho ta các tương tác chứa đạo hàm, và như vậy các đỉnh tương tác có chứa xung lượng. Quy ước chiều dương của các xung lượng là chiều đi vào đỉnh tương tác, các đỉnh tương tác loại này bao gồm: tương tác của W-boson với các Higgs (cả trung hòa và mang điện), tương tác của các Z-boson và các photon với các Higgs mang điện, tương tứng được đưa ra trong bảng 2 , bảng 3 , và bảng 4 .

Bảng 2: Các đỉnh tuơng tác của W-boson với các Higgs trung hòa và mang điện

\begin{tabular}{|l|c|}
\hline $\begin{array}{c}\text { Đỉnh tương } \\
\text { tác }\end{array}$ & Hệ số \\
\hline$h{ }_{\mu}^{ \pm} H_{1}^{\mp}$ & $\mp \frac{1}{2} g\left(\mathrm{p}_{h}-\mathrm{p}_{H_{1}}\right)_{\mu} \sin (\alpha-\beta) \sin \theta_{z}$ \\
\hline$\square_{\mu}^{ \pm} H^{\mp}{ }_{2}$ & $\pm \frac{1}{2} g\left(\mathrm{p}_{h}-\mathrm{p}_{H_{2}}\right)_{\mu} \sin (\alpha-\beta) \cos \theta_{z}$ \\
\hline$\square \square{ }_{\mu}^{ \pm} H_{1}^{\mp}$ & $\mp \frac{1}{2} g\left(\mathrm{p}_{H}-\mathrm{p}_{H_{1}}\right)_{\mu} \cos (\alpha-\beta) \sin \theta_{z}$ \\
\hline$\square \square{ }_{\mu}^{ \pm} H^{\mp}$ & $\pm \frac{1}{2} g\left(\mathrm{p}_{H}-\mathrm{p}_{H_{2}}\right)_{\mu} \cos (\alpha-\beta) \cos \theta_{z}$ \\
\hline
\end{tabular}

trong đó: $p_{h, H}$ tương ứng là xung lượng của của các Higgs trung hòa và $p_{H_{1,2}}$ là xung lượng của các Higgs mang điện.

Bảng 3: Các đỉnh tuơng tác của Z-boson với các Higgs mang điện

\begin{tabular}{|c|c|}
\hline $\begin{array}{c}\text { Đỉnh tương } \\
\text { tác }\end{array}$ & Hệ số \\
\hline$\square_{\mu} H_{1}^{ \pm} H^{\mp}{ }_{1}$ & $-g\left(\mathrm{p}_{m}-\mathrm{p}_{p}\right)_{\mu} \frac{\left(\mathrm{c}_{\square}{ }^{2}-\mathrm{s}_{\square}{ }_{\square}\right)}{2 c_{\square}} \sin ^{2} \theta_{z}$ \\
\hline$\square_{\mu} H_{2}{ }^{ \pm} H^{\mp}{ }_{2}$ & $-g\left(\mathrm{p}_{m}-\mathrm{p}_{p}\right)_{\mu} \frac{\left(\mathrm{c}_{\square}{ }^{2}-\mathrm{s}_{\square}{ }_{\square}\right)}{2 c_{\square}} \cos ^{2} \theta_{z}$ \\
\hline$\square_{\mu} H_{1,2}{ }^{ \pm} H^{\mp}{ }_{2,1}$ & $g\left(\mathrm{p}_{m}-\mathrm{p}_{p}\right)_{\mu} \frac{\left(\mathrm{c}_{\square}{ }^{2}-\mathrm{s}_{\square}{ }_{\square}\right)}{2 c_{\square}} \sin \theta_{z} \cos \theta_{z}$ \\
\hline
\end{tabular}

Bảng 4: Các đỉnh tưong tác của photon với các Higgs mang điện

\begin{tabular}{|l|c|}
\hline $\begin{array}{c}\text { Đỉnh tương } \\
\text { tác }\end{array}$ & Hệ số \\
\hline$A_{\mu} H_{1}{ }^{ \pm} H_{1}^{\mp}$ & $-g\left(\mathrm{p}_{m}-\mathrm{p}_{p}\right)_{\mu} \mathrm{s}_{\square} \sin ^{2} \theta_{z}$ \\
\hline$A_{\mu} H_{2}{ }^{ \pm} H^{\mp}{ }_{2}$ & $-g\left(\mathrm{p}_{m}-\mathrm{p}_{p}\right)_{\mu} \mathrm{s}_{\square} \cos ^{2} \theta_{z}$ \\
\hline$A_{\mu} H_{1,2}{ }^{ \pm} H^{\mp}{ }_{2,1}$ & $g\left(\mathrm{p}_{m}-\mathrm{p}_{p}\right)_{\mu} \mathrm{s}_{\square} \sin \theta_{z} \cos \theta_{z}$ \\
\hline
\end{tabular}

Trong bảng 3 và bảng 4 kí hiệu $p_{m, p}$ tương ứng là xung lượng của của các Higgs mang điện âm và Higgs mang điện dương. 
Số hạng cuối cùng của (19) cho tương tác của hai boson chuẩn với các Higgs trung hòa, hoặc tương tác của hai boson chuẩn với hai Higgs.

Bảng 5: Các đỉnh tương tác của Higgs trung hòa với các boson chuẩn

\begin{tabular}{|l|l|}
\hline \multicolumn{1}{|l|}{ Đỉnh tương tác } & Hệ số \\
\hline$h \square{ }_{\mu}{ }^{+}{ }_{v}{ }_{v}$ & $i g m_{\square} \cos (\alpha-\beta) \mathrm{g}^{\mu v}$ \\
\hline \hline$\square{ }_{\mu}{ }^{{ }^{F}}{ }_{v}$ & $i g m_{\square} \sin (\alpha-\beta) \mathrm{g}^{\mu v}$ \\
\hline$h Z_{\mu} Z_{v}$ & $\frac{g^{2} v}{4 c^{2}} \cos (\alpha-\beta) \mathrm{g}^{\mu v}$ \\
\hline$H Z_{\mu} Z_{v}$ & $-\frac{g^{2} v}{4 c^{2}} \sin (\alpha-\beta) \mathrm{g}^{\mu v}$ \\
\hline
\end{tabular}

Từ kết quả này cho thấy các Higgs trung hòa không tương tác trực tiếp với các photon, do vậy kênh rã của Higgs trung hòa ra hai photon chỉ có ở bậc bổ đính một vòng trở lên.

Bảng 6: Các đỉnh tuoong tác bậc bốn của hai Higgs với các hai boson chuẩn

\begin{tabular}{|c|c|}
\hline Đỉnh tương tác & Hệ số \\
\hline$\left(A_{\mu} ; Z_{\mu}\right) h H_{1} \square_{v}^{\mp}$ & $\frac{1}{2} g^{2} s_{\square}^{2} \sin (\alpha-\beta) \sin \theta_{z} \mathrm{~g}^{\mu v}\left(\frac{1}{s_{\square}} ; \frac{1}{c_{\square}}\right)$ \\
\hline$\left(A_{\mu} ; Z_{\mu}\right) H H_{1} \square^{\mp}$ & $\frac{1}{2} g^{2} s_{\square}^{2} \cos (\alpha-\beta) \sin \theta_{z} \mathrm{~g}^{\mu v}\left(\frac{1}{s_{\square}} ; \frac{1}{c_{\square}}\right)$ \\
\hline$\left(A_{\mu} ; Z_{\mu}\right) h H_{2} \square^{\mp}$ & $\frac{1}{2} g^{2} s_{\square}^{2} \sin (\alpha-\beta) \cos \theta_{z} g^{\mu v}\left(\frac{1}{s_{\square}} ; \frac{-1}{c_{\square}}\right)$ \\
\hline$\left(A_{\mu} ; Z_{\mu}\right) H H_{2} \square^{\mp}{ }_{v}$ & $\frac{1}{2} g^{2} s_{\square}^{2} \cos (\alpha-\beta) \cos \theta_{z} g^{\mu v}\left(\frac{1}{s_{\square}} ; \frac{-1}{c_{\square}}\right)$ \\
\hline$A_{\mu} A_{v} H_{1}^{ \pm} H_{1}^{\mp}$ & $g^{2} s_{\square}^{2} \sin ^{2} \theta_{z} g^{\mu v}$ \\
\hline$A_{\mu} A_{v} H_{2}^{ \pm} H_{2}^{\mp}$ & $g^{2} s^{2} \cos ^{2} \theta_{z} g^{\mu \nu}$ \\
\hline$A_{\mu} A_{v} H_{2}^{ \pm} H_{1}^{\mp}$ & $g^{2} s_{\square}^{2} \sin \theta_{z} \cos \theta_{z} g^{\mu v}$ \\
\hline$Z_{\mu} Z_{v} H_{1}^{ \pm} H_{1}^{\mp}$ & $g^{2} \frac{\left(c_{\square}{ }^{2}-s_{\square}^{2}\right)}{4 c_{\square}^{2}} \sin ^{2} \theta_{z} g^{\mu \nu}$ \\
\hline$Z_{\mu} Z_{v} H_{2}^{ \pm} H_{2}^{\mp}$ & $g^{2} \frac{\left(\mathrm{c}_{\square}^{2}-\mathrm{s}_{\square}^{2}\right)}{4 c_{\square}^{2}} \cos ^{2} \theta_{z} g^{\mu v}$ \\
\hline$Z_{\mu} Z_{v} H_{2}^{ \pm} H_{1}^{\mp}$ & $-g^{2} \frac{\left(c_{\Pi}^{2}-s_{\square}^{2}\right)}{4 c_{\square}^{2}} \cos \theta_{z} \sin \theta_{z} g^{\mu \nu}$ \\
\hline
\end{tabular}

Các đỉnh tương tác của các boson chuẩn có các đặc điểm riêng biệt như sau: $i$ ) các đỉnh tương tác có fermion luôn đi kèm với ma trận Dirac $\left(\gamma^{\mu}\right)$, ii) các đỉnh tương tác có chứa đạo hàm của các trường luôn kèm theo xung lượng, iii) các đỉnh tương tác có chứa hai boson luôn gắn liền với tensor hạng $2\left(g^{\mu v}\right)$.

\section{Kết luận.}

Trong phạm vi mô hình Zee, bài báo đạt được các kết quả sau:

- Xác định trạng thái vật lý của các boson chuẩn, các Higgs boson trung hòa và mang điện. Đặc biệt, với các fermion cần đưa vào các ma trận chuyển cơ sở $\left(U_{a b}, V_{a b}\right)$ để liên hệ giữa cơ sở ban đầu và cơ sở khối lượng (cơ sở ứng với các trạng thái vật lý).

- Đưa ra các đỉnh tương tác của các boson chuẩn với các fermion.

- Đưa ra các đỉnh tương tác bậc ba và bậc bốn của các boson chuẩn và các Higgs boson (cả trung hòa và mang điện).

Các đỉnh tương tác này là cơ sở để xác định các giản đồ Feynman cũng như các kênh rã khác nhau của các quá trình rã của Higgs boson. Các tín hiệu thu được từ các quá trình rã Higgs boson sẽ góp phần đưa ra các hiện tượng Vật lý mới.

\section{TÀI LIỆU THAM KHẢO}

1. Hoàng Ngọc Long (2006), Co sở Vật lý hạt cơ bản, NXB Thống kê, Hà Nội.

2. A.Zee (1980), A theory of lepton number violation and neutrinos Majorana masses, Phys. Lett. Vol.93B, No.4,.

3. ATLAS and CMS Collaborations, Measurements of the Higgs boson production and decay rates and constraints on its couplings from a combined ATLAS and CMS analysis of the LHC pp collision data at $\sqrt{s}_{\mathrm{s}}=7$ and $8 \mathrm{TeV}$, JHEP 1608 (2016) 045.

4. C. Patrignani et al. [Particle Data Group], Review of particle physics, Chinese Physics C 40 (2016) 100001.

5. E. Misuda, K. Sasaki (2001), Zee model and phenomenology of lepton sector, YNU-HEPTh-01-102.

6. M.Cheng,K.Cheung, Zee model and Neutrinoless double beta decay, NSC-NCTS 020304 (2002).

7. M.E.Peskin, D.V.Schroeder (1995), An introduction to quantum fields theory, Westview press.

8. R. Longas, D. Portillo, D. Restrepo, O. Zapata (2016), , The Inert Zee Model, JHEP 03,162.

9. X.He, S.K. Majee (2012), Implications of Recent Data on Neutrino Mixing and Lepton Flavour Violating Decays for the Zee Model, JHEP 1203, 023. 